\title{
Entrelacs
}

Cinéma et audiovisuel

$6 \mid 2007$

L'Arbre

\section{Excroissances du séquoïa de Vertigo dans La Jetée et L'Armée des 12 singes}

Sophie Lecole-Solnychkine et Arnaud Despax

\section{(2) OpenEdition}

Journals

Édition électronique

URL : http://journals.openedition.org/entrelacs/323

DOI : 10.4000/entrelacs.323

ISSN : 2261-5482

Éditeur

Éditions Téraèdre

Édition imprimée

Date de publication : 1 mars 2007

Pagination : 59-69

ISBN : 978-2-912868-70-1

ISSN : 1266-7188

Référence électronique

Sophie Lecole-Solnychkine et Arnaud Despax, «Excroissances du séquoïa de Vertigo dans La Jetée et L'Armée des 12 singes », Entrelacs [En ligne], 6 | 2007, mis en ligne le 01 août 2012, consulté le 19 avril 2019. URL : http://journals.openedition.org/entrelacs/323 ; DOI : 10.4000/entrelacs.323

Ce document a été généré automatiquement le 19 avril 2019

Tous droits réservés 


\title{
Excroissances du séquoïa de Vertigo dans La Jetée et L'Armée des 12 singes
}

\author{
Sophie Lecole-Solnychkine et Arnaud Despax
}

« L'image est ce en quoi le maintenant rencontre

l'autrefois,

en une fulgurance, pour former une constellation neuve

Walter Benjamin

1 Profitant de l'apparente quiétude d'une après-midi ensoleillée, un homme et une femme se promènent dans un parc. Au fil des sentiers, à travers les arbres, leurs pas les conduisent devant l'installation, destinée aux promeneurs, d'une coupe d'arbre, sur les cernes duquel figurent des dates importantes de l'Histoire. Dans trois films, nous avons repéré que cette séquence, bien qu'appartenant à des trames narratives différentes, se répète quasiment à l'identique. L'intériorité dévoilée de l'arbre met au jour un vaste réseau de résonances, faisant ainsi germer de l'arbre mort, au travers de ses nœuds, un ensemble de ramifications, de boutures, de greffons, développant une complexe arborescence reliant les trois œuvres. Ce motif de l'arbre, repéré dans des films où il tient un rôle diégétique essentiel, formera le prétexte d'une interrogation sur les potentialités interprétatives de l'œuvre ${ }^{1}$, à la faveur d'une herméneutique entendue non pas comme recherche d'un sens, d'une vérité, mais d'un tissu pluriel d'éléments signifiants, comme autant de bourgeons, d'excroissances de l'arbre source.

2 Vertigo/Sueurs froides d'Alfred Hitchcock (1958), La Jetée de Chris Marker (1962), Twelve Monkeys/L'armée des 12 singes de Terry Gilliam (1995) : ces films, qui s'articulent autour de phénomènes manifestes d'intertextualité et de citations, voire de réécriture, sont tous trois reliés par la présence d'un arbre, avec cette particularité capitale qu'il s'agit d'un arbre tronqué. La section d'un tronc de séquoia millénaire (dont la matrice se trouve dans Vertigo), prend ainsi une résonance essentielle en s'inscrivant au cœur d'une réflexion sur la mémoire, le souvenir, le rêve, l'histoire collective et l'histoire personnelle, le temps global et le temps raconté. 
3 La citation la plus explicite de notre corpus filmique se trouve dans L'armée des 12 singes (1997) : après la scène du kidnapping du père de Jeffrey par les improbables militants de l'« armée des 12 singes ", subitement, de façon non motivée, et en plein écran, débute une séquence prélevée telle quelle dans Vertigo et qui correspond à la visite de Madeleine et John au Sequoia National Park (Californie). Ce n'est qu'au cours des plans suivants qu'est relié à la diégèse l'emprunt direct à Hitchcock : nous nous trouvons dans une salle de cinéma dans laquelle les deux protagonistes assistent à la projection du film.

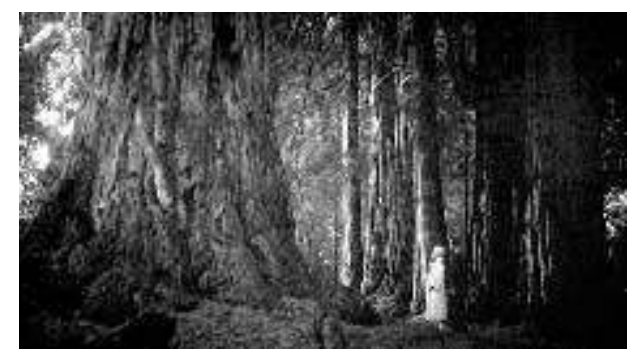

4 Cette citation vient radicaliser les enjeux des échos de Vertigo dans La Jetée, principale inspiration du film de Gilliam, ainsi que l'indique le générique². Bamchade Pourvali confirme ce lien intertextuel : "À travers la coupe de séquoia du Jardin des plantes à Paris, La Jetée se rattache à Vertigo. C'est à " Hitchcock » que fait allusion le " nom étranger " prononcé par la femme. Mais d'autres similitudes témoignent de la relation entre les deux films : les tombes, le profil droit d'Hélène Chatelain. ${ }^{3}$ " Ces remarques invitent à une étude comparative entre les scènes de l'arbre des deux films sources de Twelve Monkeys : Vertigo et La Jetée. Afin de faciliter la lecture, précisons tout d'abord le contexte narratif de ces deux scènes.

5 Le héros de Vertigo (1958), John Ferguson, souffrant d'acrophobie, est contraint de cesser son activité de détective. Un ami, Elster, lui demande un jour de suivre sa femme Madeleine car il s'inquiète des troubles psychiques qu'il a remarqués chez elle; elle semble souffrir d'aliénation mentale, et s'identifier à une femme qui s'est suicidée depuis longtemps, Carlotta Valdes. Après l'avoir sauvée de la noyade, John tombe amoureux de Madeleine, mais ne peut l'empêcher de connaître le même sort que le prétendu fantôme : elle se jette du haut d'un clocher. Par la suite, rencontrant une femme qui ressemble étrangement à Madeleine, John comprendra qu'il a été la victime impuissante du stratagème d'Elster, voulant tuer sa femme et ayant trouvé en la personne de John le témoin idéal du meurtre déguisé en suicide. Au moment où a lieu la scène se déroulant au Parc National, Madeleine et John ont décidé de joindre leurs solitudes et se promènent dans une forêt.

6 La Jetée, photo-film de 1962, montre un Paris post-apocalyptique où les survivants d'une guerre sans précédent en sont réduits à survivre sous terre. Des scientifiques tentent, par l'expérimentation du voyage temporel sur des prisonniers, d'établir un pont avec une autre époque afin de trouver une solution à leur impasse. Le héros, l'un des cobayes, est expédié dans le passé où il rencontre une jeune femme, dont le visage le hante depuis toujours, image au statut incertain de souvenir, de rêve, voire de fantasme (cet argument constituera le canevas de L'armée des 12 singes). 


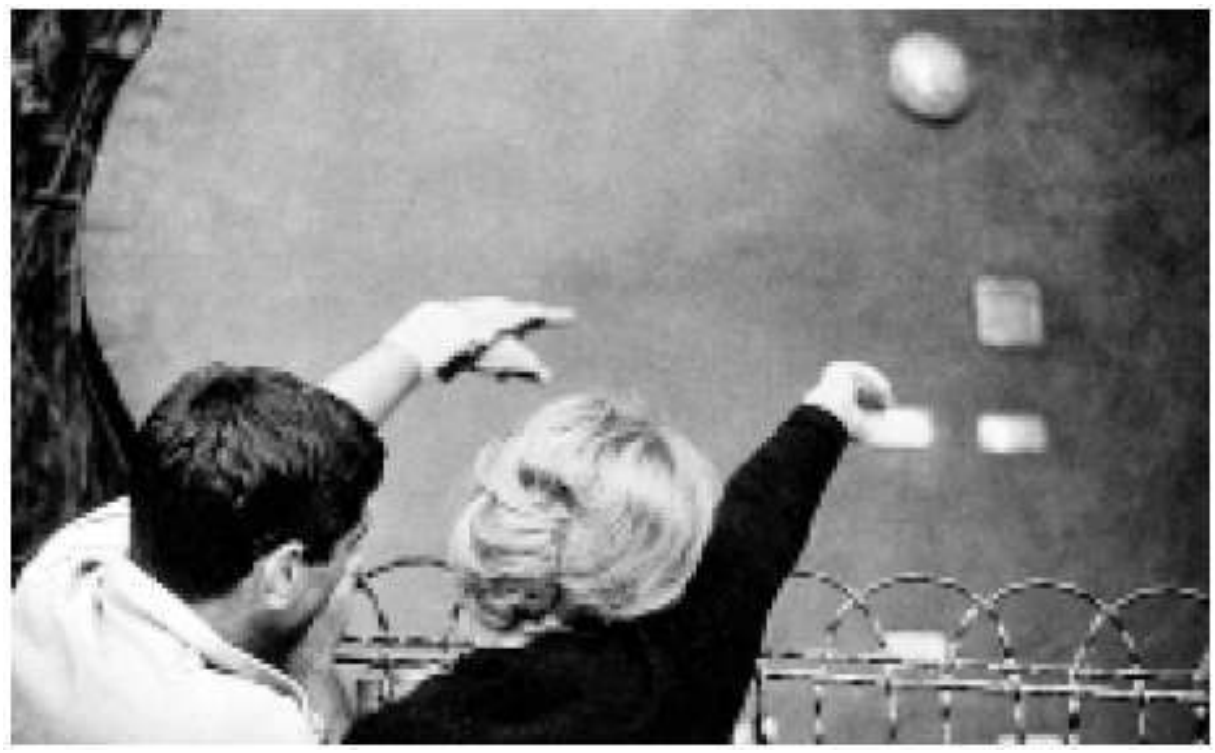

7 Dans l'un de ses voyages vers cette femme qu'il retrouve régulièrement, l'homme se promène avec elle au Jardin des plantes, dans un Paris d'avant-guerre. Après quelques plans de déambulation, apparaît une image où l'homme matérialise un espace entre ses deux mains : on comprend à la faveur du plan qui suit (qui montre le même geste des mains en contrechamp) qu'il s'agit d'une portion de la coupe de l'arbre devant laquelle ils se trouvent, donc d'une portion de temps : est-ce le temps d'une vie, de sa vie, ou le temps qui les sépare lui et elle?

Alors que la musique composée par Trevor Duncan laisse entendre des accents manifestement repris de celle de Bernard Herrman pour Vertigo, la voix du récitant, en off , poursuit :

Ils s'arrêtent devant une coupe de séquoia couverte de dates historiques. Elle prononce un nom étranger qu'il ne comprend pas. Comme en rêve, il lui montre un point en dehors de l'arbre. Il s'entend dire : "Je viens de là ", et y retombe, à bout de forces.

Dans les deux films, un couple de personnages, un homme et une femme, entre lesquels vont se tisser des liens affectifs assez forts, se promènent, le hasard de leurs pas les menant face à une coupe de séquoia sur laquelle des dates ont été inscrites. La motivation des deux scènes est identique, c'est-à-dire précisément très faible au regard de l'intrigue. Ces personnages désignent d'un geste des points sur la coupe : désigner un point sur le bois, c'est ainsi indiquer un instant, une période. La matérialité végétale de l'arbre devient pure temporalité, inscription vertigineuse du temps humain dans le temps de l'arbre. L'impression, auparavant fugace, de disproportion entre l'existence humaine et le temps naturel se dévoile, jusqu'à devenir tactile, gravée dans la chair même de l'arbre, touchée du doigt par les protagonistes songeurs. À Madeleine qui s'enquiert de l'âge de l'un des arbres présents alentour, John répond : « Oh... deux mille ans, si ce n'est plus. » Madeleine pense à « tous les gens qui sont nés et qui sont morts pendant que ces arbres continuaient à vivre. »

- John : Leur nom savant est sequoia sempervirens : toujours vert, toujours vivant.

- Madeleine : Je ne les aime pas.

- John : Pourquoi?

- Madeleine : Parce que je dois mourir.

10 On peut également établir un rapprochement entre les apparitions et disparitions successives du héros de La Jetée, dues à ses voyages dans le temps, et la « disparition » de 
Madeleine derrière un tronc dans la forêt. Cette dernière apparaît comme une métaphore de la dissolution de l'individu dans l'altérité : tout se passe comme si l'on avait affaire à un moment où la disparition apparaît, où la perte des repères spatiaux et temporels, dans la forêt californienne profonde et sombre, correspond à la perte de soi. Ce motif sera d'ailleurs radicalisé dans Twelve Monkeys, lorsque James Cole (Bruce Willis) disparaît dans la forêt, en une véritable dissipation physique, puisqu'il est rappelé dans le futur, dans le temps qui est le sien. Il est intéressant de souligner le lien qui semble émerger entre les figures de la perte de soi, de la perte de repères spatiaux et temporels, et l'égarement dans le bois. Mais si la silhouette de Madeleine disparaît pour un instant seulement, cachée par l'un des troncs gigantesques dans une forêt aux sentiers balisés, domestiquée voire touristique, chez Gilliam Cole disparaît pour de bon dans une vraie forêt, sauvage, hostile. Quoiqu'il en soit, cette scène et ses reprises remotivent une thématique pour le moins classique, celle de l'égarement dans le bois, et la forêt devient théâtre de la dissolution de l'individu dans des temps et des espaces sur lesquels il n'a aucune prise.

Enfin, tout comme Madeleine semble subir l'envahissement de son identité par celle d'une morte, le héros de La Jetée vit cette expérience " comme en rêve ». Au-delà de cette thématique de la confusion psychique, commune aux deux films, s'étendent les ombres de la folie, mais surtout de la manipulation : le discours de Madeleine est en réalité commandé par le stratagème de son pseudo-mari Elster qui veut se débarrasser de sa vraie femme, tandis que ce sont les vainqueurs de la guerre qui, dans La Jetée, envoient le héros en mission dans le passé. Objectalisés, voire réifiés, les deux personnages deviennent les instruments d'une volonté supérieure et obscure.

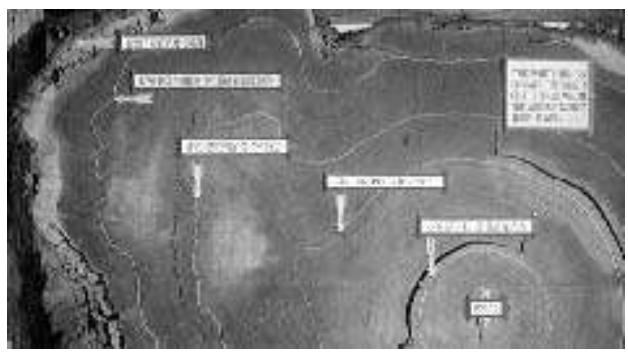

Il n'échappe pas que l'on assiste pour ce dernier point à une inversion des rôles entre les sexes. Cela étant, les attitudes respectives de John et de la jeune femme de La Jetée sont très différentes, l'un étant soumis à l'obsession de "trouver la clef » de l'énigme, et l'autre au contraire ne posant aucune question. D'autre part, à la forêt de Californie s'est substitué le Jardin des plantes de Paris. Dès lors, si la forêt peut symboliser pour Madeleine le risque de la folie, c'est-à-dire de la perte de soi au travers de la perte de repères, le Jardin des plantes de La Jetée évoque plutôt la quête de repères par un héros venu du futur et voulant trouver dans le passé une solution au désastre. Paradoxalement, alors qu'un plan frontal permet de lire le détail des dates inscrites sur le tronc dans Vertigo, aucune image ne les montre dans La Jetée. Enfin, la différence capitale entre ces deux séquences consiste en la variation du propos des personnages : Madeleine dit en désignant successivement deux endroits des cernes : « Je suis née quelque part par ici, et je suis morte là. Pour lui ça n'a été qu'une heure... Pour moi une vie. " Cela semble signifier que la vie de l'arbre contient dans son intégralité la « vie » de l'héroïne, dans la mesure où son discours est prétendument celui de la psyché de Carlotta Valdes. Le héros de La Jetée, quant à lui, « comme en rêve, [...] montre un point en dehors de l'arbre » et s'entend dire " Je viens de là ". Remarquons que l'effet fantastique de cette phrase ne vient pas tant de l'extériorité de la vie du locuteur par rapport à celle de l'arbre (il a pu en 
effet naître indifféremment avant ou après sa coupe), que du mélange du temps et de l'espace. " Je viens de là » : non seulement les verbes naître et mourir se changent en un verbe de mouvement, mais encore l'adverbe là, déictique de l'espace, devient déictique du temps. Ce qui est signifié ici n'est rien de moins que la spatialisation temporelle qu'implique le voyage dans le temps. Il n'est pas anodin que, lorsque ses geôliers essaient de l'envoyer dans le temps, le héros se trouve confronté à la coupe d'un arbre, qui marque elle aussi la spatialisation du temps. Ce point qu'il montre en dehors de l'arbre, est un point inexistant et qui n'existera jamais car l'arbre, mort, débité en tronçons, a cessé de croître. Désigner l'invisible, c'est pour l'homme la seule et unique manière de se faire comprendre, du point de vue du voyage temporel. Par conséquent, ces mots font de l'arbre la figure du hic et nunc, de l'ici et du maintenant, du centre d'où rayonne le réseau signifiant de l'œuvre, ce que l'emplacement de ce fragment corrobore : exactement au milieu du film. Cette situation médiane, qui confère à l'arbre une importance structurale, se retrouve dans les autres films du corpus. En effet, la séquence au National Parc se situe presque à la moitié du film dans Vertigo. Dans Twelve Monkeys, cette séquence intervient plutôt sur la fin du film. D'un point de vue chronologique, cette scène de la confrontation à l'arbre, au Temps, qui leur est commune, se déplace donc au fil des œuvres : très légèrement antérieure au centre pour la première, à l'exact milieu pour la seconde, plutôt vers la fin pour la troisième.

Comme nous l'avons noté plus haut, la séquence extraite de Vertigo et insérée dans Twelve Monkeys arrive de façon abrupte. Il semble toutefois qu'une transition puisse se lire entre la séquence dans la camionnette des " 12 singes ", où Jeffrey (Brad Pitt) est traité de fou par son père, et l'apparition de Kim Novak à l'écran, dont le personnage présente comme nous l'avons vu des signes d'instabilité mentale. La folie serait alors ce qui relie ces séquences, nous faisant également passer du singe à l'arbre.

Le travail de la citation effectué ainsi par Gilliam - explicite dans ce cas, contrairement à ce qu'on a dans La Jetée - fonctionne selon le mode d'un montage alterné qui manifeste l'identité de destin entre les deux couples. En effet, Madeleine et Cole sont des personnages au passé flou, qui vont tous deux rencontrer une seconde personne prête à les aider à discerner le rêve du souvenir, le fantasme de la réalité, le vrai du faux. Cette similitude est sursignifiée dans le passage suivant, où la citation ${ }^{4}$ est présentée comme un souvenir d'enfance :

- Cole : Il me semble que j'ai déjà vu ce film quand j'étais petit, à la télé,je l'ai déjà vu.

- Kathryn : Chut, tais-toi.

-John : Êtes-vous déjà venue ici ? Quand êtes-vous née?

- Cole, troublé : Je ne reconnais pas.

- John : Madeleine, dites-moi...

- Cole : Exactement comme pour nous, comme le passé. Le film est toujours le même, il ne

change pas. Et à chaque vision il semble différent parce qu'on est différents. On le voit différemment.

Auparavant, rappelons que les protagonistes en fuite, James Cole, l'envoyé du futur, et sa psychiatre Kathryn Railly (Madeleine Stowe), avaient acheté des vêtements dans un grand magasin. Est ainsi explicitée la motivation de cette séquence : ils ont besoin d'un endroit discret pour revêtir leurs déguisements, ce que leur offre la pénombre du cinéma. Si Kathryn ne prête pas attention au film, Cole le regarde pendant qu'elle s'affaire à le grimer. La concomitance entre les phrases décousues de Cole et l'enjeu sémantique du travestissement est extrêmement pertinente, dans la mesure où Cole justement déclare : "Le film est toujours le même, il ne change pas ", au moment où Kathryn lui étale de la 
colle sous le nez afin de lui mettre une moustache, pour précisément essayer de le changer. Quoique affublé en outre d'une perruque, il sera toujours le même, il ne changera pas, et l'issue tragique du film non plus, en accord avec ce que le souvenir (de l'individu personnage et de l'œuvre source) configure dès le départ.

Or, cette thématique du souvenir est essentielle dans l'intrigue de La Jetée dont la première phrase, prononcée par le récitant et inscrite en plein texte, est la suivante : "Ceci est l'histoire d'un homme hanté par un souvenir d'enfance ». Si Cole commence ici à percevoir qu'il se souvient réellement de Kathryn, c'est qu'il reconnaît le film d'Hitchcock, qui resurgit de sa mémoire avec le caractère flou du souvenir. Il comprend enfin l'origine de ce sentiment qui l'habite, qui est d'avoir toujours connu Kathryn : c'est la jeune femme qu'il a vue enfant dans un aéroport - à l'intérieur du bâtiment et non sur la jetée comme dans le film de Marker. Ce point est révélateur d'une double prise de distance par rapport au film source : non seulement Gilliam modifie légèrement le lieu du souvenir, mais encore, au lieu de citer directement le passage du Jardin des plantes, il remonte à la citation supposée initiale, celle de Vertigo, qui était implicite dans La Jetée. Cet effet de boucle permet d'homogénéiser la matière cinématographique, tout en soulignant le travail de re-création.

Il est un fait que la figure de l'arbre préside aussi à un complexe réseau de relations citationnelles entre les trois films : arborescence où la toile sémantique se tisse de phénomènes de répétitions, de reprises, d'altérations, de transformations, d'inversions, de substitutions, dessinant au fil des œuvres un espace parallèle, transversal aux trois films, où ces trois séquences s'interpénètrent, comme autant de ramifications, de boutures, de greffons, croissant et proliférant sur ce sol nouveau.

L'utilisation habile du montage alterné (entre les deux séquences montrées en champcontrechamp) insère progressivement les enjeux sémantiques de Vertigo dans la diégèse de Twelve Monkeys. Plus encore, ce sont les dialogues mêmes de Vertigo qui guideront, ou même orienteront l'action dans Twelve Monkeys : on parvient ainsi à une intrication complexe, à un entrelacement des répliques des deux couples de protagonistes, Kathryn/ James et Madeleine/John :

- Madeleine : Promettez-moi de ne plus de poser de questions, promettez-le moi.

- Cole : Kathryn... Pourquoi fais-tu tout ça ?... Je veux savoir... Et si je me trompais. Si tu te

trompais. Si j'étais vraiment fou.

- John : Je suis responsable de vous maintenant.

- Cole : Que veux-tu faire?

- John : Il faut que je sache

$-[\ldots]$

- Kathryn : Tu m'as dit que tu n'as jamais vu l'océan.

Dans ce passage, la phrase de Madeleine vient paradoxalement enclencher une série d'interrogations formulées par Cole, qui, influencé par ce qu'il voit et entend de Vertigo, se suppose fou, au moment même où Kathryn, mettant entre parenthèses sa fonction de psychiatre, commence à croire au bien-fondé des assertions de Cole. Croire en la vérité de son histoire motive un ensemble d'actions impliquant la responsabilité de la jeune femme envers l'homme au moment même où John dans Vertigo endosse la responsabilité de la vie de Madeleine. La phrase de Cole : " Je veux savoir... », annonce celle de John : « Il faut que je sache ", en un subtil jeu d'entremêlement sémantique. L'image suivante, montrant Kim Novak et James Stewart au bord de la mer, motive d'autre part la réplique finale de Kathryn, dite après qu'elle a tourné pour la première fois les yeux vers l'écran. Notons l'échange dialectique des attitudes, puisqu'au début de la scène, ce sont les réflexions de 
Cole qui naissent de la vision du film : c'est là le moment précis où la diégèse de Vertigo pénètre celle de Twelve Monkeys pour l'orienter. De simple motif, la reprise devient prétexte, argument.

Cet échange participe du thème de l'inversion, qui se réalise entre les films notamment au niveau de l'interversion des sexes, ainsi que nous l'évoquions précédemment: la confusion des femmes dans Vertigo devient celle des hommes chez Gilliam et Marker. Le rôle de celui qui essaie de reconstituer le passé, de démêler le rêve du souvenir, assumé dans un premier temps par John, l'est ensuite par Kathryn, tandis que la femme de La Jetée se contente d'accueillir le voyageur sans l'interroger. La Madeleine de Vertigo est folle, ou plutôt se présente théâtralement comme telle, et John, l'ayant sauvée d'un premier suicide, choisit de l'aider, assumant ainsi la responsabilité de son existence (« Je suis responsable de vous maintenant »). Dans Twelve Monkeys, c'est le personnage masculin qui se croit fou, et c'est à sa comparse qu'incombe cette même responsabilité morale. La scène analysée ci-dessus constitue ainsi le climax de cette prise de conscience, de cet engagement moral envers l'autre.

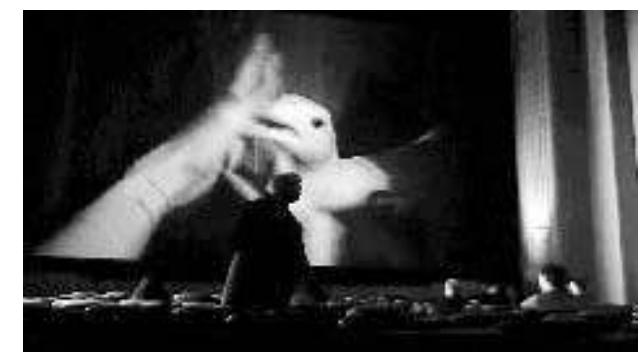

21 Le changement de couleur de cheveux consécutif au travestissement des héros des 12 singes relève du même jeu de substitution : de brune, Kathryn devient blonde, présentant ainsi une ressemblance troublante non seulement avec Kim Novak dans Vertigo ${ }^{5}$, mais aussi avec Hélène Chatelain dans La Jetée. En sortant du cinéma, qui proposait une rétrospective Hitchcock, c'est un Cole hésitant, troublé, qui aperçoit Kathryn grimée : "Je ne t'avais pas reconnue ", « Oui, moi non plus, tu as beaucoup changé toi aussi ». Ce dialogue se déroule sur fond de musique de Vertigo, bien que logiquement ce soit celle du film Les Oiseaux que nous devrions entendre ; en effet, nous avons pu voir précédemment quelques séquences facilement identifiables de cet autre film, projeté à l'écran lorsque Cole, qui s'était endormi dans la salle de cinéma, s'y réveille, grimé lui aussi. C'est que le changement se produit sur fond de similitude, pour mieux présider à la reconnaissance des motifs rémanents qui circulent d'une œuvre à l'autre :

- Cole : Ça a toujours été toi, dans mon rêve c'était toi.

- Kathryn : Et je me souviens de toi comme ça. J'avais l'impression de te connaître, j'ai

l'impression de t'avoir toujours connu.

- Cole : J'ai peur...

Ces mots de Cole, alors que tous deux s'enlacent, font écho à ceux de Madeleine, qui, serrée dans les bras de John, lui avoue : "J'ai si peur ». Mais si désormais Kathryn est blonde, si elle porte un imperméable beige, ayant ainsi revêtu tous les signes de l'héroöne hitchcockienne, elle n'a cependant pas les cheveux attachés, à la différence de Madeleine qui porte un chignon caractéristique ${ }^{6}$, en spirale, lié au thème de l'acrophobie ainsi que l'exploite intelligemment le générique de Saul Bass. Les cheveux blonds, lâchés, identifient également Kathryn à la jeune femme de La Jetée, cette femme aux cheveux volant dans le vent de la jetée d'Orly, cette femme du souvenir d'enfance qui sera reconnue à l'heure de la mort. S'affirme ainsi un jeu subtil de ressemblance/ 
dissemblance, d'identité/altérité, de reprise du thème avec variation, selon un mode d'altération du motif itératif. Le réinvestissement par Gilliam de deux films célèbres lui permet dès lors d'exploiter les possibilités autoréflexives du cinéma, en mettant à profit le travestissement des deux protagonistes dans une salle obscure pour enclencher une réflexion sur le travail de transformation et de réappropriation que produit le film. Il semble que cette mise en abyme suggère la capacité de dissimulation/révélation du cinéma : le film, en tant qu'œuvre d'art, avance masqué, révèle en dévoilant à demi mots. La conclusion de cette séquence donne par conséquent un éclairage nouveau aux propos de Cole : « Le film est toujours le même, il ne change pas. Et à chaque vision il semble différent parce qu'on est différents. On le voit différemment " : à travers la singularité d'une œuvre se dessine l'homogénéité à la fois foncière et artificielle de toute la production cinématographique, qui connaît l'unification grâce à la conscience d'un réalisateur apte à en faire jaillir les enjeux représentationnels.

Empreint des œuvres de son passé de cinéphile, Terry Gilliam actualise ainsi les schèmes artistiques des films d'Hitchcock et de Marker, les projetant dans un à-venir du cinéma, qui, du repiquage au marcottage, possède tous les traits d'une arborescence.

Même le fortuit se voit alors susceptible d'être interprété : il faut dire ici le phénomène piquant de correspondance altérée entre les noms des acteurs et des personnages : Madeleine est certes le prénom du personnage de Kim Novak dans Vertigo ; c'est aussi celui de l'actrice qui joue Kathryn Railly dans Twelve Monkeys, Madeleine Stowe. Et si James Stewart joue John dans Vertigo, le personnage de Bruce Willis dans Twelve Monkeys s'appelle James Cole. Y aurait-il ici jeu conscient et sophistiqué de la part du réalisateur, démiurge des sens ? Quoi qu'il en soit au fil des films et des visions, du couple MadeleineJames demeure l'écho sans cesse repris d'une figure, " qui porte absence et présence, plaisir et déplaisir ${ }^{7}$ ", dans la mémoire, dans l'œuvre, et dans le temps.

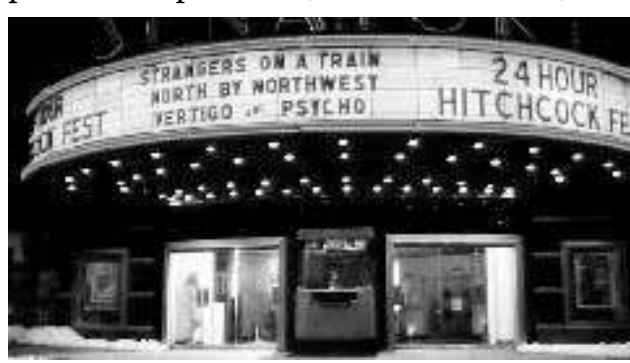

Au Crépuscule des Dieux, le Wotan de Wagner abat le frêne cosmique Yggdrasil et en dispose les bûches autour du Wallhala en vue de l'incendie universel. Au cinéma, la coupe de l'arbre, fruit de la violence, ne débouche pas nécessairement sur une catastrophe ; au contraire, elle constitue un ensemble de repères, un nœud de significations (les nœuds du bois ?) propice à suggérer la relation entre les œuvres et les temps.

Abattre l'arbre, et le débiter, c'est pour l'œuvre filmique la possibilité de spatialiser la temporalité cachée du végétal, en mettant au jour par la mort la totalité d'une vie, en manifestant la mobilité du temps afin de rendre possible une interrogation herméneutique à son égard. Ce temps qui fait croître l'arbre est aussi le soubassement du film ; en tronquant l'arbre, le film l'intègre de force dans une temporalité spatiale (tout comme une horloge est la manifestation spatiale et divisée du flux indivisible du temps), pour offrir du même coup aux personnages l'opportunité d'une réflexion sur leur mémoire et leur histoire, et au spectateur celle d'une réflexion sur l'art même du film, son esthétique, son langage, sa rhétorique. En fait, le thème de l'arbre tronqué tel qu'il se 
présente chez Hitchcock, Marker et Gilliam, jetant de telles passerelles entre les films, nous a invité à rien moins que déchiffrer les modalités du rapport interdiscursif d'une œuvre à une autre. Il n'est dès lors pas inconcevable de penser que si le film fait cesser l'invisible développement de l'arbre vivant, c'est au profit d'une résurrection de son pouvoir symbolique de correspondances : décontextualisation (le temps se fait espace), et recontextualisation (le temps spatialisé désigne l'horizon temporel), ces deux opérations conceptuelles étant très exactement celles du travail de la citation, exemplaire du rapport intertextuel.

Une fois l'arbre coupé, le film, l'intégrant en son sein, se substitue à lui en proposant de manière citationnelle un ensemble de ramifications et d'embranchements, de greffons, de boutures : l'intertexte fonctionne comme une arborescence. Le jeu de mise en abyme de l'intertextualité cinématographique insère l'arbre dans une esthétique de l'altération : le plaisir artistique, naissant de l'intervalle entre reconnaissance d'un motif connu et conscience de la différence à travers sa transformation, dépasse ici le tragique des destins. le maintenant rencontre l'autrefois, en une fulgurance, pour former une constellation neuve ».

\section{NOTES}

1. Nous entendons par là non seulement la pluralité d'interprétations que l'œuvre appelle, mais également l'éclairage qu'elle peut apporter sur d'autres œuvres avec lesquelles elle entretient un rapport intertextuel.

2. «Inspired by the film La Jetée written by Chris Marker ».

3. Bamchade POURVALI, Chris Marker, Paris, Éditions Cahiers du Cinéma, Les petits Cahiers, SCÉRÉN-CNDP, 2003. L'auteur précise en bas de page la source de cette comparaison : « Le texte du film dans L'Avant-Scène cinéma, n 38, du 15 avril 1964, porte comme note le "nom étranger »: «Hitchcock». En 1992, le livre bilingue La Jetée, aux éditions «Zone Books» cite le nom agrémenté, toutefois, d'un point d'interrogation ».

4. Afin de faciliter la compréhension des passages de films cités, où les dialogues des protagonistes de Vertigo et de ceux de Twelve Monkeys sont entremêlés, les dialogues du couple John-Madeleine figureront en gras.

5. Qui d'ailleurs sera travestie à nouveau en Madeleine, re-teinte en blonde, lorsque redevenue Judy, elle subit les métamorphoses que lui impose John.

6. Rappelons qu'il s'agit aussi du chignon de Carlotta Valdes, personnage qui a prétendument pris possession de son identité.

7. Blaise Pascal, Pensées. 


\section{AUTEURS}

\section{SOPHIE LECOLE-SOLNYCHKINE}

Doctorante - Allocataires Moniteurs

Laboratoire de Recherche en Audiovisuel

Equipe Littérature et Herméneutique

Université de Toulouse II Le Mirail

\section{ARNAUD DESPAX}

Doctorant - Allocataires Moniteurs

Laboratoire de Recherche en Audiovisuel

Equipe Littérature et Herméneutique

Université de Toulouse II Le Mirail 